\title{
Electrical properties of improper ferroelectrics from first principles
}

\author{
Massimiliano Stengel, ${ }^{1,2}$ Craig J. Fennie, ${ }^{3}$ and Philippe Ghosez ${ }^{4}$ \\ ${ }^{1}$ ICREA - Institució Catalana de Recerca i Estudis Avançats, 08010 Barcelona, Spain \\ ${ }^{2}$ Institut de Ciència de Materials de Barcelona (ICMAB-CSIC), Campus UAB, 08193 Bellaterra, Spain \\ ${ }^{3}$ School of Applied 83 Engineering Physics, Cornell University, Ithaca, NY 14853 US \\ ${ }^{4}$ Physique Théorique des Matériaux, University of Liège, 4000 Liège, Belgium
}

\begin{abstract}
We study the interplay of structural and polar distortions in hexagonal $\mathrm{YMnO}_{3}$ and short-period $\mathrm{PbTiO}_{3} / \mathrm{SrTiO}_{3}$ superlattices by means of first-principles calculations at constrained electric displacement field $D$. We find that in $\mathrm{YMnO}_{3}$ the tilts of the oxygen polyhedra produce a robustly polar ground state, which persists at any choice of the electrical boundary conditions. Conversely, in PTO/STO the antiferrodistortive instabilities alone do not break inversion symmetry, and opencircuit bundary conditions restore a non-polar state. We suggest that this qualitative difference naturally provides a route to rationalizing the concept of "improper ferroelectricity" from the point of view of first-principles theory. We discuss the implications of our arguments for the design of novel multiferroic materials with enhanced functionalities, and for the symmetry analysis of the phase transitions.

PACS numbers: 71.15.-m,77.84.-s,77.55.Nv
\end{abstract}

\section{INTRODUCTION}

Multifunctional oxide materials, characterized by coexisting ferroic orders of different physical origin, are currently the object of intense exploration. Particularly enticing for their potential technological applications and fundamental interest are the so-called multiferroic materials, where spontaneous magnetic and ferroelectric orders coexist in the same phase. Unfortunately, few multiferroic oxides are known, and their magnetoelectric coupling is usually too small (or occurs at exceedingly lower temperatures) for practical purposes. This is typically explained as follows: in the most popular ferroelectric oxides, such as $\mathrm{BaTiO}_{3}$, the spontaneous polarization emerges from the hybridization between occupied $\mathrm{O}(2 p)$ orbitals and empty $3 d$ orbital of the transitionmetal cation. This $d^{0}$-ness condition typically rules out magnetism. Although exceptions have been identified ${ }^{1,2}$, ferroelectricity and magnetism often appear to be contraindicated $\mathrm{3}^{\underline{3}}$

One way to achieve a strong coupling between electrical and magnetic degrees of freedom, is to resort to a more restrictive class of materials, called "improper ferroelectrics", where the polarization is induced by completely different microscopic mechanisms. $\stackrel{4}{*}$ (Another less restrictive mechanism involves the idea of phase competition ${ }^{5,6}$.) Strictly speaking, the distinction between proper and improper ferroelectrics is made on symmetry grounds, and is based on the dynamics of the phase transition from a high-temperature centrosymmetric to a lower-temperature polar phase. In proper ferroelectrics (PF), such as $\mathrm{PbTiO}_{3}$ or $\mathrm{BaTiO}_{3}$, the polar distortion acts as the primary order parameter in the phase transition; conversely, in improper ferroelectrics (IF) the polarization $P$ is slave to another, non-polar, order parameter of different physical origin through an energy term linear in $P$. Depending on the material these can be the rotation/tilts of the oxygen polyhedra network, or the mag- netic order associated, e.g. with spin cycloids. Unlike covalency-driven polar distortions, these alternate mechanisms can potentially lead to strong magnetoelectric couplings. For example spin-driven improper ferroelectricity in $\mathrm{TbMnO}_{3}{ }^{7}$ naturally couples magnetic and polar degrees of freedom, resulting in a remarkable magnetoelectric effect, while structurally-driven improper ferroelectricity in $\mathrm{YMnO}_{3}{ }^{8}$ leads to an unusual locking of polar and antiphase structural domain walls ${ }^{9}$. These recent discoveries suggest an opportunity to achieve entirely new classes of strongly coupled multifunctional materials with novel properties based on improper ferroelectricity. As we said above, however, improper ferroelectrics are not abundant in nature. As a result material design rules have remained elusive and the discovery of new multiferroics occurs serendipitously.

This situation is now rapidly changing thanks to the increasing popularity of density-functional theory calculations, which have led the quest for new oxide materials in the past few years. An important breakthrough in the field 10 occurred by the recent discovery of a new type of improper ferroelectricity in artificially layered $\mathrm{PbTiO}_{3} / \mathrm{SrTiO}_{3}(\mathrm{PTO} / \mathrm{STO})$ superlattices. This system displays an unusual form of lattice-driven instability, involving the interplay of $P$ with octahedra rotations and tilts. More specifically, the free energy of this system was shown to display an unusual trilinear coupling, $\sim \Phi_{1} \Phi_{2} P$, between the polarization and two non-polar antiferrodistortive (AFD) modes of different symmetry, $\Phi_{1}$ and $\Phi_{2}$. This work has paved the way towards the rational design of lattice-driven improper ferroelectrics 11 with a large spontaneous $P$ and has lead to the discovery of a novel class of $\left(\mathrm{CaMnO}_{3}\right)_{2} \mathrm{CaO}$ strongly coupled multiferroics $\frac{12}{2}$. Given the versatility of perovskites, this novel class of IFs are ripe systems to explore for new multifunctional phenomena.

There are however important questions that still need to be answered from the point of view of fundamental 
theory. For example, the origin of the polarization is not always clear if in addition to the two AFD instabilities - which from the trilinear coupling could lead to a $P \sim \Phi_{1} \Phi_{2}$ - a proper ferroelectric instability exists. This issue is ultimately tied to the question of which two order parameters can be identified as inducing the distorted state (e.g., in this case $\Phi_{1} \sim \Phi_{2} P$ is an equally valid statement). This question of primary order parameters determines to what extent the electrical and dielectric properties behave like conventional IFs. This is of central importance for the applicability of multifunctional materials in actual devices. Unfortunately the ability to clearly address this using symmetry arguments and/or conventional first-principles calculations alone is difficult. What is clearly missing is a generally applicable and reliable "test" within a zero-temperature calculation, which would unambiguously identify whether the properties of this class of (hybrid) IFs behaved more like a conventional IF or more like a proper ferroelectric.

Here we compare from first principles the electrical properties of hexagonal $\mathrm{YMnO}_{3}$ to that of the PTO/STO superlattice. We illustrate the key fingerprints of improper ferroelectricity by using a recent advance in firstprinciples methods ${ }^{13}$ that treats the electrical displacement, $D$, as the fundamental variable. This allows us to directly compute the spontaneous polarization in opencircuit, $P_{\mathrm{OC}}$, within a periodic bulk calculation, avoiding the need for cumbersome slab geometries (where the results inevitably depend on the details of the surfaces; a common source of artifacts in the calculation). We identify the presence of a nonzero and switchable $P_{\mathrm{OC}}$ as a necessary (although generally not sufficient) condition that must be satisfied by a material in order for it to be classified as improper ferroelectric. This property is enjoyed by $\mathrm{YMnO}_{3}$ (albeit with a substantially smaller spontaneous $P_{\mathrm{OC}}$ than reported previously), but not by the $1 / 1 \mathrm{PbTiO}_{3} / \mathrm{SrTiO}_{3}$ superlattices considered here. We rationalize this unexpected result in terms of higher order couplings between the AFD mode(s) and the polarization. We briefly discuss the implications of these result for the future design of novel improper ferroelectric materials. This approach puts the concept of improper ferroelectricity on firmer conceptual grounds from the point of view of ab-initio theory, providing a useful tool for the rational design of new "hybrid" 12 IFs.

\section{COMPUTATIONAL METHODS}

Our calculations are performed within the localdensity approximation (LDA) of density-functional theory and the projector-augmented-wave (PAW) method ${ }^{14}$, with a plane-wave cutoff of $60 \mathrm{Ry}$. For $\mathrm{YMnO}_{3}$, we apply a Hubbard correction $(\mathrm{LDA}+\mathrm{U})$ of $\mathrm{U}=6 \mathrm{eV}$ to the Mn $3 d$ states, in order to obtain an insulating ground state. We further assume a collinear antiferromagnetic configuration of the spins, and use a $4 \times 4 \times 2$ MonkhorstPack $^{15}$ (MP) sampling of the 30-atom hexagonal unit
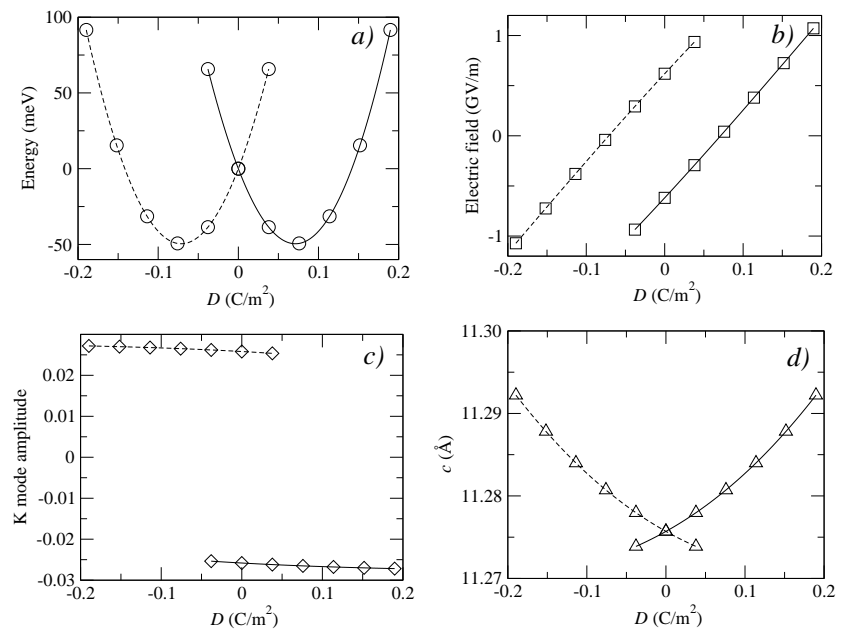

FIG. 1: Electrical properties of $\mathrm{YMnO}_{3}$. The energy (a), internal electric field (b), $K_{3}$ mode amplitude (c) and out-ofplane strain (d) are plotted as a function of the macroscopic $D$ field. Symbols refer to first-principles data; the continuous lines are SPLINE fits to the symbols.

cell to perform the Brillouin-zone integrals. We use standard non-spin polarized LDA for PTO/STO, together with a $4 \times 4 \times 3 \mathrm{MP}$ grid to sample the Brillouin zone of the $\sqrt{2} \times \sqrt{2} \times 2$ direct-space cell (20 atoms total, doubled in-plane to accommodate the antiferrodistortive rotations of the oxygen octahedra). Cell relaxations are performed by using the calculated stress tensor, which we correct for the Pulay error by applying a constant negative pressure. For $\mathrm{YMnO}_{3}$, first we optimize the structure of the ferroelectric phase, and then fix the inplane lattice parameter to the equilibrium value in zero applied field. For PTO/STO we fix the in-plane lattice parameter to the equilibrium theoretical value of cubic $\mathrm{SrTiO}_{3}$, as it has became common practice in simulations of layered perovskites. (We relax only the out-of-plane lattice parameter in response to the electrical perturbations, as typically these materials are grown as epitaxial films on a thick substrate, which prevents their in-plane relaxation.) The macroscopic electrical degrees of freedom are dealt with by using the methods described in Ref. 13. In all cases we enforce rather stringent threshold for structure relaxations, as the polarization and the internal electric fields are both very sensitive to minimal displacements of the ions. More specifically, we calculate the ground state of both materials as a function of the macroscopic electric displacement field, and analyze the results in terms of energy, internal electric field, amplitude of the relevant structural (zone-boundary) modes and out-of-plane (electrically induced) strain. 


\section{RESULTS}

\section{A. $\mathrm{YMnO}_{3}$}

In a proper ferroelectric, open-circuit boundary conditions suppresses single-domain ferroelectricity and drives domain formation. Imposing open-circuit boundary conditions corresponds to adding to the free energy a positive term that is quadratic in $P^{13}$,

$$
\Delta E=\frac{\Omega}{8 \pi}(D-4 \pi P)^{2},
$$

where $\Omega$ is the volume and $D$ is the electric displacement of the high-temperature paraelectric phase, which can be taken as zero. Remarkably, Sai et al. 16 demonstrated by means of first-principles calculations that a monodomain state with non-zero $P$ can persist in $\mathrm{YMnO}_{3}$ even in the extreme limit of an unsupported film without electrodes. Because the polarization couples linearly to the primary order parameter in an improper ferroelectric $\stackrel{4}{ }$, any IF must enjoy such a property.

So are there clearly identifiable features in the electrical diagram that distinguish an improper ferroelectric from that of a proper ferroelectric? In Figure 1(a) we plot the energy as a function of the electric displacement field for $\mathrm{YMnO}_{3}$. As expected from a ferroelectric compound, there are two symmetric potential wells, whose minima correspond to a spontaneous polarization of $P_{\mathrm{s}}=7.1$ $\mu \mathrm{C} / \mathrm{cm}^{2}$. This is slightly larger than previously reported values ${ }^{8,16}$; we ascribe the small difference to the different pseudopotentials used, and to the different (real-space) approach used here to compute the macroscopic polarization 17 . Remarkably, the electrical equation of state is a multi-valued function in the region near the origin. Moreover, the curves belonging to the two symmetrical wells cross each other in $D=0$, rather than converging smoothly into a saddle point. Both features are in stark contrast to the known behavior of "standard" ferroelectric materials that display a proper ferroelectric transition such as $\mathrm{PbTiO}_{3}$ or $\mathrm{BaTiO}_{3}$.

This is not completely unexpected, as the physical origin of ferroelectricity in $\mathrm{YMnO}_{3}$ differs from that of $\mathrm{BaTiO}_{3} . \mathrm{YMnO}_{3}$ is a classic improper ferroelectric, in which the spontaneous $P$ is driven by an unstable, nonpolar "trimerization" $\left(K_{3}\right)$ mode such that $P \propto K_{3}^{3}$. Thus, rather than destabilizing the polarization, the $K_{3}$ distortion pushes the zone-center polar modes away from their centrosymmetric equilibrium configuration, generating a polarization in the process. In other words, the $K_{3}$ mode acts like a switchable "geometric field" 8 that, depending on its orientation, produces two inequivalent (but related by a mirror symmetry operation) states of the system with opposite tendency towards a polar distortion. Either state shows an almost perfectly linear dielectric behavior [see Figure1(b), the electric field follows an almost perfect straight line], with an average dielectric constant $\bar{\epsilon}_{33} \sim 13$, and an extremely weak piezoelectric response, of the order of $d_{33} \sim 1 \mathrm{pm} / \mathrm{V}$. Note that

\begin{tabular}{ccccc}
\hline \hline & $\mathrm{FE}_{z}$ & $\mathrm{AFD}_{\mathrm{zi}}$ & $\mathrm{AFD}_{\mathrm{zo}}$ & $(\mathrm{AFD} / \mathrm{FE})_{z}$ \\
\hline$P_{z}$ & 30 & 0 & 0 & 32 \\
$\phi_{\mathrm{zo}}$ & 0 & 0 & 5.4 & 4.6 \\
$\phi_{\mathrm{zi}}$ & 0 & 3.3 & 0 & 3.1 \\
$\Delta E$ & -12 & -4 & -32 & -56 \\
\hline \hline
\end{tabular}

TABLE I: Properties of the relevant phases in PTO/STO. The spontaneous polarization $P_{z}$ is in $\mu \mathrm{C} / \mathrm{cm}^{2}$; the angles $\phi$ are in degrees; energies $\Delta E$ (referred to the $P 4 / \mathrm{mmm}$ phase) are in $\mathrm{meV}$.

the amplitude of the $\mathrm{K}_{3}$ phonon mode does not change much across the whole range of $D$ values, $\max \pm 5 \%$ with respect to the ferroelectric ground state. This is a consequence of the relatively weak coupling (only third-order) between the $\mathrm{K}_{3}$ mode and $P$, and to the comparatively high energy associated to the $K_{3}$ structural transition, which is more than three times higher than the highest electrostatic energy, about $0.15 \mathrm{eV}$, obtained in our simulations. In passing, we note that a similar kink in the electrical equation of state at $P=0$ was previously reported for another ferroelectric material, $\mathrm{KNO}_{3}$, where there is also a structural mode that produces a bistable state in the origin 18,19 . We identify this characteristic kink, which is a consequence of the above-mentioned "geometric field", as the ab-initio fingerprint of improper ferroelectricity.

Our calculations indeed show, in agreement with Ref. 16 that there is a non-zero polarization in open circuit, $P_{\mathrm{OC}}$, which corresponds to the $D=0$ point in the electrical diagrams of Figure [1] Since $D=0$, $P_{\mathrm{OC}}=-\mathcal{E}_{\mathrm{OC}} / 4 \pi$, where $\mathcal{E}_{\mathrm{OC}}=-0.62 \mathrm{GV} / \mathrm{m}$ is the internal field; this yields $P_{\mathrm{OC}}= \pm 0.55 \mu \mathrm{C} / \mathrm{cm}^{2}$. (The ratio between the spontaneous polarizations in short circuit and open circuit corresponds to the average dielectric constant of $\mathrm{YMnO}_{3}$ within this region of the electrical diagram: $P_{\mathrm{s}} / P_{\mathrm{OC}}=\bar{\epsilon}_{33} \sim 13$.) Note that these values depart significantly from those $\left(P_{\mathrm{OC}}=6.1 \mu \mathrm{C} / \mathrm{cm}^{2}\right.$, $\mathcal{E}_{\mathrm{OC}}=0.25 \mathrm{GV} / \mathrm{m}$ ) reported in Ref. 16. To account for this discrepancy, we can speculate that the unsupported films of Ref. 16 are not under ideal open-circuit boundary conditions: The metallic oxygen-terminated surfaces would act effectively as electrodes, that partially screen the polarization charges of $\mathrm{YMnO}_{3}$. This highlights the importance of the analysis introduced in this work for understanding the intrinsic properties of improper ferroelectrics. We shall come back to this point in Section IIIC, where we show how one can predict, from the calculated bulk data alone, the behavior of a film under any type of electrical boundary conditions, ranging from open-circuit to close-circuit, and including all intermediate "imperfect screening" 20 regimes. 

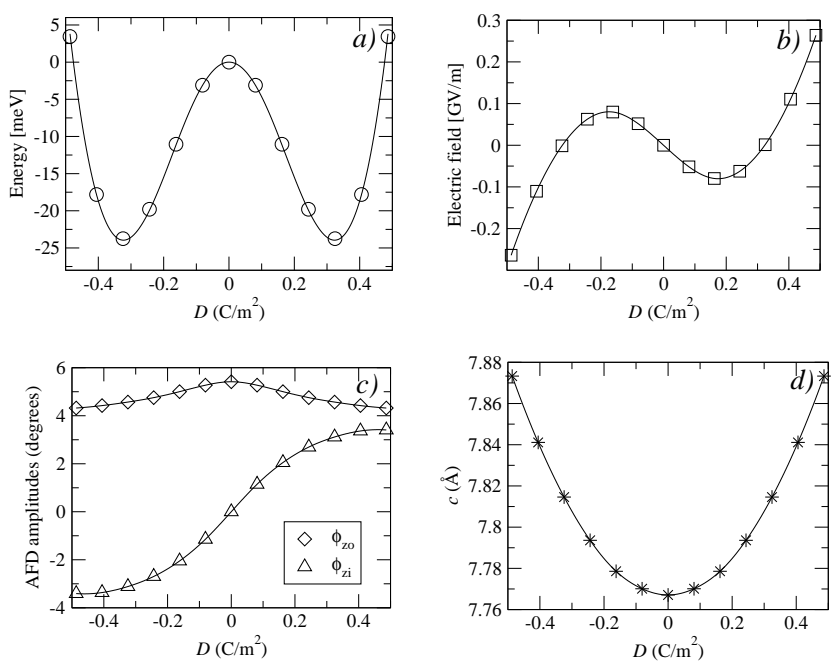

FIG. 2: Electrical properties of the PTO/STO superlattice. The energy (a), internal electric field (b), AFD mode amplitudes (c) and out-of-plane strain (d) are plotted as a function of the macroscopic $D$ field.

\section{B. $\mathrm{PbTiO}_{3} / \mathrm{SrTiO}_{3}$ superlattices}

We now consider the PTO/STO superlattice. In the high-symmetry $P 4 / \mathrm{mmm}$ reference phase we find three unstable distortion modes, in agreement with Ref. 10; a zone-center polar mode, plus two symmetryinequivalent AFD rotations of the oxygen octahedrals along the $z$ axis, either out of phase $\left(\mathrm{AFD}_{\mathrm{zo}}\right)$ or in phase $\left(\mathrm{AFD}_{\mathrm{zi}}\right)$. (Note that we restrict our analysis to modes with rotation/displacement axis along $z$.) By freezing in each of these instabilities separately and then performing full structural relaxations we obtain three new phases of lower symmetry labeled $\mathrm{FE}_{z}, \mathrm{AFD}_{z i}$, and $\mathrm{AFD}_{z o}$ in Table [1. Furthermore, we obtain a fourth phase, $(\mathrm{AFD} / \mathrm{FE})_{z}$, in which all three instabilities coexist. While we find some discrepancies at the quantitative level between our results and that of Ref. 10, the level of qualitative agreement confirms their main conclusion. The energy gains associated to ferroelectric transitions $\left[P 4 / m m m \rightarrow \mathrm{FE}_{z}, \mathrm{AFD}_{\mathrm{zo}} \rightarrow(\mathrm{AFD} / \mathrm{FE})_{z}\right]$ are nevertheless systematically larger by a factor of 3-4 here (respectively -3 and $-8 \mathrm{meV}$ in Ref. 10, to be compared with -12 and $-56+32=-24 \mathrm{meV}$ in Table 【), while the $\mathrm{AFD}_{z i}$ and $\mathrm{AFD}_{z o}$ instabilities appear systematically less pronounced (-28 and $-73 \mathrm{meV}$ in Ref. 10). Ferroelectric perovskites are known to be especially sensitive to the computational approximations and these differences can be traced back in the different pseudopotentials used in both calculations and in the subsequent different in-plane lattice constant at which the calculations have been performed.

The large energy gains associated with the ferroelectric transition that we calculate suggests in fact that the polarization might be one of the primary order parameters (the other being $\mathrm{AFD}_{\mathrm{zo}}$ ) rather than the two rotation

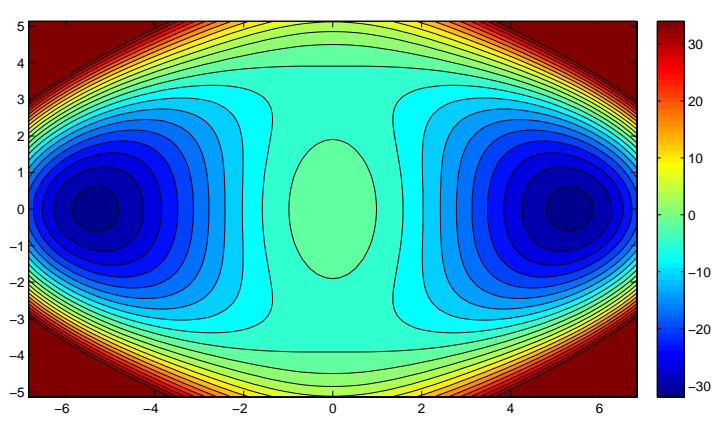

FIG. 3: Two-dimensional energy landscape of the AFD modes in the PTO/STO superlattice around the $P 4 / \mathrm{mmm}$ reference structure. Horizontal $\left(\phi_{z o}\right)$ and vertical $\left(\phi_{z i}\right)$ axes are the mode amplitudes in degrees. The unit of the color bar is meV.

distortions. Applying the approach outlined in this work should clarify this point. In Figure 2 we summarize the non-linear electrical and structural response of the superlattice as a function of $D$, analogous plots as in Figure 1. Unlike $\mathrm{YMnO}_{3}, \mathrm{PTO} / \mathrm{STO}$ appears to behave in all respects like a standard ferroelectric material. The doublewell potential in Figure 2(a) is a single-valued function, differentiable at the saddle point, qualitatively similar to typical soft-mode driven systems like $\mathrm{PbTiO}_{3}$ and $\mathrm{BaTiO}_{3}$. This was to some extent unexpected : recent reports of improper ferroelectricity on this same system suggested that its properties might closely mirror those of $\mathrm{YMnO}_{3} \underline{10}$. To gain insight into our apparently contradictory results, it is useful to look at the response of the AFD modes - which interact nontrivially with the ferroelectric polarization - to an applied $D$-field. In Figure 2(c) we show the amplitude of the out-of-phase $\mathrm{AFD}_{\mathrm{zo}}$ and of the in-phase $\mathrm{AFD}_{\mathrm{zi}}$ modes as a function of $D$. At $D=0$, only a pure $\mathrm{AFD}_{\mathrm{zo}}$ distortion is present (i.e., this configuration is identical to the pure $\mathrm{AFD}_{z o}$ phase reported in Table I), while the $\mathrm{AFD}_{\mathrm{zi}}$ distortion and the polarization are absent $\left(\mathrm{AFD}_{z o}\right.$ is a centrosymmetric space group.) This explains why we do not observe a linear behavior of the energy around $D=0$ in Figure 2(a). Only when we move away from $D=0$ do we see a linear increase of $\mathrm{AFD}_{\mathrm{zi}}$, the signature of the tri-linear coupling discussed in Ref. 10 .

To illustrate why the $\mathrm{AFD}_{\mathrm{zo}}$ and $\mathrm{AFD}_{\mathrm{zi}}$ do not coexist at $D=0$, we performed a series of calculation where we freeze in various amplitudes of either mode, starting from the relaxed superlattice in the reference $P 4 / \mathrm{mmm}$ symmetry. In Figure 3 we plot the energy as a function of the two independent amplitudes $\left(\phi_{\mathrm{zo}}, \phi_{\mathrm{zi}}\right)$ within the restricted two-dimensional subspace spanned by these modes. As speculated above, both modes are unstable in the reference structure, but the condensation of either mode quickly suppresses the instability with respect to the other (consistent with the results of Ref $\underline{\underline{10}}$ ). The global minima of the two-dimensional energy land- 


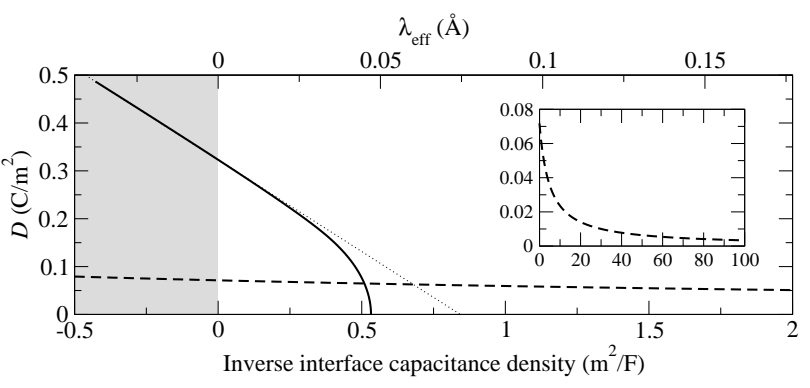

FIG. 4: Equilibrium $D$ for a thin-film PTO/STO (solid line) or $\mathrm{YMnO}_{3}$ (dashed line) capacitor as a function of $\lambda_{\text {eff }} / N$ or $\mathcal{C}_{\mathrm{I}}^{-1} / N$. The shaded area refers to a hypothetical overscreening regime with $\lambda_{\text {eff }}<0$. The dotted line is a guide to the eye, to highlight the linear behavior of $D$ in PTO/STO for $\lambda_{\text {eff }} / N<0.025 \AA$. The inset shows the absence of "critical thickness" in $\mathrm{YMnO}_{3}$ in the limit of large $\lambda_{\text {eff }} / N$.

scape corresponds to pure $\mathrm{AFD}_{\mathrm{zo}}$ states (the out-of-phase instability is much stronger than the in-phase one), in agreement with the $D=0$ ground state found in our electrical diagram of Figure 2(a).

In the following Section we further substantiate these points by comparing the performance of hypothetical $\mathrm{PTO} / \mathrm{STO}$ - or $\mathrm{YMnO}_{3}$-based capacitors with real electrodes (i.e. under imperfect screening conditions).

\section{From bulk to thin-film capacitors}

So far we have only discussed the bulk electrical properties of $\mathrm{YMnO}_{3}$ and short-period PTO/STO superlattices. However, for practical applications in thin-film capacitors, it is necessary to take possible finite size effects into account. In particular, the imperfect screening at a realistic film/electrode interface is known to affect the stability of the ferroelectric state by altering the electrical boundary conditions of the film $\underline{20,21}$. In the following we shall demonstrate that the impact of such interface effects can be directly predicted from the bulk equations of state of Fig. 1] and 2, i.e. without doing explicitly a demanding calculation of an actual capacitor.

We assume two symmetric electrode/ferroelectric interfaces, which behave as two thin layers of linear dielectric interposed between the insulator and the metal. These can be characterized indifferently by an interface capacitance density per unit area $\mathcal{C}_{\mathrm{I}}$, or by an effective screening length $\lambda_{\text {eff }}=\epsilon_{0} / \mathcal{C}_{\mathrm{I}}{ }^{21}$. Then, the electrical equation of state of such a device can be written $\operatorname{as}^{22}$

$$
U(D)=N U_{\mathrm{Bulk}}(D)+2 \frac{S D^{2}}{2 \mathcal{C}_{\mathrm{I}}}
$$

where $U_{\text {Bulk }}$ is the energy as in Fig. 1(a) and 2(a); $S$ is the cell cross-section and $N$ is the thickness of the film expressed in number of bulk units. The minimum of $U(D)$ within short-circuit boundary conditions (zero applied potential) is given by the stationary point $d U / d D=0$ (recall that the potential $V$ is related to $U$ by $\left.S V(D)=d U / d D^{22}\right)$,

$$
V(D)=N V_{\text {Bulk }}(D)+\frac{2 D}{\mathcal{C}_{\mathrm{I}}}=0 .
$$

This can be written more compactly in terms of the interfacial "effective screening length" $\lambda_{\text {eff }}=\epsilon_{0} / \mathcal{C}_{\text {I }}$ as

$$
\frac{\lambda_{\mathrm{eff}}}{\epsilon_{0} N}=-\frac{V_{\mathrm{Bulk}}(D)}{2 D} .
$$

By inverting Eq. 4 we can predict the behavior of a thin film of arbitrary thickness $N$ and with an arbitrary $\lambda_{\text {eff }}$ (the ground state depends on $\lambda_{\text {eff }} / N$ only).

In Fig. 4(a) we plot the resulting equilibrium $D$ of a hypothetical $N=1$ capacitor as a function of $\lambda_{\text {eff }}$. (We also consider small negative values of $\lambda_{\text {eff }} / N$ to account for a possible overscreening 21.23.) PTO/STO (solid line) shows the typical destabilization of the ferroelectric state (critical thickness for ferroelectricity) under an imperfect screening regime; this occurs at $\lambda_{\text {eff }} / N=0.047 \AA$. Conversely, $\mathrm{YMnO}_{3}$ appears almost unsensitive to the electrical boundary conditions, with a spontaneous polarization that persists up to arbitrarily large values of $\lambda_{\text {eff }} / N$. The limit $\lambda_{\text {eff }} / N \rightarrow \infty$ corresponds to open-circuit boundary conditions, where $D$ tends to zero asymptotically as $\sim N / \lambda_{\text {eff }}$.

\section{From first principles to Landau theory}

While our first-principles results yield a fairly detailed picture on the electrical behavior of the compounds under consideration, it is very useful to recast these data in terms of a simplified physical model, where the role played by the active degrees of freedom emerges clearly. Also, most researchers in the ab-initio community are used to analyzing the behavior of ferroelectrics by performing calculations under zero external field, where the energy landscape as function of the active structural distortions is explored by freezing in various amplitudes of selected imaginary-frequency phonons. In this Section we shall trace a formal link between the constrained- $D$ method used in this work and the more familiar understanding of ferroelectrics, within either Landau theory or the frozen-phonon approach.

Consider the zero-field first-principles Kohn-Sham total energy, written as a function of the electronic degrees of freedom $\psi$ and the ionic positions $u$ (we omit the explicit discussion of the strain to avoid complicating the notation), $E_{\mathrm{KS}}(\{\psi\},\{u\})$. Around the high-symmetry configuration, one can construct a harmonic expansion in terms of the $N$ lattice eigenmode amplitudes $\xi_{i}$,

$$
E_{\mathrm{KS}}(\{\xi\}) \sim \alpha_{1} \xi_{1}^{2}+\ldots+\alpha_{N} \xi_{N}^{2},
$$

with the assumption that all $\xi_{i}$ vanish in the reference structure. The polarity of the phonons can be expressed via the mode effective charge, $Z_{i}=\Omega d P / d \xi_{i}$ (we assume 
transversal zone-center modes, and we restrict the analysis to one component of the polarization only). Assume that the lattice distortions do not significantly affect the purely electronic dielectric constant, $\epsilon_{\infty}$, which we consider linear in the applied external field; then we can readily write the constrained- $D$ functional

$$
U(\{\xi\}, D)=E_{\mathrm{KS}}(\{\xi\})+\frac{\Omega}{2 \epsilon_{0} \epsilon_{\infty}}\left(D-P_{\xi}\right)^{2},
$$

where $P_{\xi}$ is the lattice-mediated contribution to the polarization,

$$
P_{\xi}=\frac{1}{\Omega}\left(\xi_{1} Z_{1}+\ldots \xi_{N} Z_{N}\right) .
$$

The ground state at a given $D$ is then obtained by taking the global minimum with respect to all degrees of freedom,

$$
U(D)=\min _{\xi_{i}} U(\{\xi\}, D)
$$

Now, to make progress from here it is useful to note that not all phonon modes are equally important to describe the material. Most modes are hard, with little polar activity. Only few low-energy phonons display a marked anharmonic behavior or are unstable; these are the modes that we wish to focus our attention on. Typically, the hard modes are discarded from the Hamiltonian, or better, are incorporated into an effective background constant; conversely, the low-energy modes are kept explicit, and higher-order terms are introduced in the Hamiltonian to ensure their proper description. Assume for example that only one mode, $\xi_{s}$, is "soft", and that it can be reasonably well described by a quadratic plus a quartic term,

$$
E_{\mathrm{KS}}\left(\xi_{s}\right)=\alpha_{s} \xi_{s}^{2}+\beta \xi_{s}^{4} .
$$

Assume also that the phonon-phonon interaction is small, and that the electronic susceptibility is not significantly affected by $\xi_{s}$. Then the constrained- $D$ functional can be simplified by taking the minimum with respect to the hard modes only,

$$
\begin{aligned}
U\left(\xi_{s}, D\right) & =\min _{\xi_{i}, i \neq s} U(\{\xi\}, D) \\
& =\alpha_{s} \xi_{s}^{2}+\beta \xi_{s}^{4}+\frac{\Omega}{2 \epsilon_{0} \epsilon_{\mathrm{B}}}\left(D-P_{s}\right)^{2} .
\end{aligned}
$$

Here we have introduced the background dielectric constant $\stackrel{24}{\rightleftharpoons} \epsilon_{\mathrm{B}}$, which is given in terms of $\epsilon_{\infty}$, the (hard) mode charges $Z_{i}$ and their eigenfrequencies $\omega_{i}$,

$$
\epsilon_{\mathrm{B}}=\epsilon_{\infty}+\sum_{i \neq s} \frac{1}{\Omega} \frac{Z_{i}^{2}}{\omega_{i}^{2}}
$$

Open-circuit boundary conditions correspond to setting $D=0$ in the above equation. In such a regime it is easy to see that the electrostatic energy term in $U$ is just an additional quadratic (and positive) term in the soft mode amplitude, of the type $\Delta \xi_{s}^{2}$. Typically, this additional term completely suppresses the ferroelectric instability: $\alpha_{s}+\Delta>0$.

The above discussion establishes an important result: if we know the approximate low-energy Hamiltonian in zero field, the "background permittivity" and the effective charges of the active modes (all these ingredients can be indifferently extracted from $a b$ initio calculations or from a phenomenological analysis) we can immediately calculate the ground state at a specific value of $D$. In other words, the so-called constrained- $D$ method is by no means specific to $a b$ initio calculations, and can be applied just as well to effective Hamiltonians and phenomenological descriptions. Indeed, mapping the properties of a given material in "electric displacement space" $\underline{25}$ may be a powerful tool to validate second-principles or macroscopic approaches against the microscopic firstprinciples theory - if the descriptions are of the same quality, the results should coincide. In the following we discuss how these ideas apply to our two testcases, $\mathrm{YMnO}_{3}$ and $\mathrm{PTO} / \mathrm{STO}$.

In $\mathrm{YMnO}_{3}$ the low-energy Hamiltonian can be written as a function of a polar (but not soft) $\Gamma$ mode and by an unstable $K$-point mode,,$\underline{8}$

$$
\begin{aligned}
E_{\mathrm{KS}}\left(\xi_{\Gamma}, \xi_{K}\right) & \sim \alpha_{\Gamma} \xi_{\Gamma}^{2}+\alpha_{K} \xi_{K}^{2}+ \\
& +\beta_{K K} \xi_{K}^{4}+\beta_{\Gamma K} \xi_{\Gamma}^{2} \xi_{K}^{2}+\gamma \xi_{\Gamma} \xi_{K}^{3}
\end{aligned}
$$

The electrical variables have little impact on the structural $K$-point mode, that acts like a "switch" (see Fig. 1), and can therefore be approximated by a constant with \pm sign,

$$
E_{\mathrm{KS}}\left(\xi_{\Gamma}\right) \sim \alpha_{\Gamma} \xi_{\Gamma}^{2}+\tilde{\beta} \xi_{\Gamma}^{2} \pm \tilde{\gamma} \xi_{\Gamma} .
$$

Typical values of the parameters have been reported by Fennie and Rabe $\underline{\underline{8}}$. The biquadratic coupling renormalizes the $\Gamma$-mode frequency by further hardening it - the new quadratic coefficient is given by $\tilde{\alpha}=\alpha_{\Gamma}+\tilde{\beta}$. The last term is responsible for the geometric field. If we plug this Hamiltonian into the electrostatic energy functional we obtain

$$
U\left(\xi_{\Gamma}, D\right)=\tilde{\alpha} \xi_{\Gamma}^{2} \pm \tilde{\gamma} \xi_{\Gamma}+\frac{\Omega}{2 \epsilon_{0} \epsilon_{\mathrm{B}}}\left(D-P_{\Gamma}\right)^{2},
$$

where $P_{\Gamma}=Z_{\Gamma} \xi_{\Gamma} / \Omega$. This is a second-order polynomial that can be solved analytically for the ground state at a given $D$,

$$
U(D)=\frac{\Omega}{2 \epsilon_{0} \epsilon_{\mathrm{TOT}}}\left(D \pm P_{0}\right)^{2}
$$

where $\epsilon_{\mathrm{TO}}$ is the total dielectric constant, including the contribution from the $\Gamma$ mode, and $P_{0}$ is the spontaneous polarization of $\mathrm{YMnO}_{3}$ in zero field. This equation accurately describes the results shown in Fig. [1] where $\mathrm{YMnO}_{3}$ emerges from the calculations as a linear dielectric with $\epsilon_{\mathrm{TOT}} \sim 13$ and a switchable polarization 
$P_{0}=7.1 \mathrm{C} / \mathrm{m}^{2}$. In particular, if we impose open-circuit boundary conditions by setting $D=0$, the electric field is

$$
\mathcal{E}_{\mathrm{OC}}=\left.\frac{d U}{d D}\right|_{D=0}= \pm \frac{P_{0}}{\epsilon_{0} \epsilon_{\mathrm{TOT}}}
$$

and the polarization is $P_{\mathrm{OC}}=-\epsilon_{0} \mathcal{E}_{\mathrm{OC}}=\mp P_{0} / \epsilon_{\mathrm{TOT}}$, consistent with our analysis of the first-principles data.

The case of PTO/STO is slightly more complicated, due to the presence of three active modes instead of two, but we can follow a similar line of reasoning. The basic physics can be understood in terms of three phonon modes of the high-symmetry $P 4 / \mathrm{mmm}$ reference structure: a polar $\Gamma$ mode $\xi_{\Gamma}$, and two zone-boundary structural modes that correspond to out-of-phase and in-phase rotations of the oxygen octahedra with respect to the $z$ axis, $\phi_{z o}$ and $\phi_{z i}$. The energy in zero field can be expanded as

$$
\begin{aligned}
E_{\mathrm{KS}}\left(\xi_{\Gamma}, \phi_{z o}, \phi_{z i}\right) & =A_{\Gamma} \xi_{\Gamma}^{2}+B_{\Gamma \Gamma} \xi_{\Gamma}^{4}+ \\
& +A_{o} \phi_{z o}^{2}+B_{o o} \phi_{z o}^{4}+ \\
& +A_{i} \phi_{z i}^{2}+B_{i i} \phi_{z i}^{4}+ \\
& +B_{o \Gamma} \phi_{z o}^{2} \xi_{\Gamma}^{2}+B_{i \Gamma} \phi_{z i}^{2} \xi_{\Gamma}^{2}+B_{o i} \phi_{z o}^{2} \phi_{z i}^{2}+ \\
& +C \phi_{z o} \phi_{z i} \xi_{\Gamma}
\end{aligned}
$$

The first three lines correspond to the double-well potential associated with each individual mode $\left(\alpha_{\Gamma}, \alpha_{z o}, \alpha_{z i}<\right.$ 0 ); the fourth line provides the lowest-order biquadratic couplings responsible for the pair-wise competition or cooperation between the modes; the last line is the tri-linear coupling, that was previously identified as key to a novel type of improper ferroelectricity in this system. As the rotations do not directly contribute to $P$ (their dynamical charge is zero), the simplified constrained- $D$ functional can be written as

$U\left(\xi_{\Gamma}, \phi_{z o}, \phi_{z i}, D\right)=E_{\mathrm{KS}}\left(\xi_{\Gamma}, \phi_{z o}, \phi_{z i}\right)+\frac{\Omega}{2 \epsilon_{0} \epsilon_{\mathrm{B}}}\left(D-P_{\Gamma}\right)^{2}$

At $D=0$, the electrostatic energy term reduces to $\gamma \xi_{\Gamma}^{2}$ with $\gamma$ a positive constant; this effectively renormalizes the $A_{\Gamma}$ coefficient to $\tilde{A}_{\Gamma}=A_{\Gamma}+\gamma$. Recall that $A_{\Gamma}$ is negative - in our ab initio model PTO/STO has a ferroelectric instability even in absence of rotations. Conversely, $\tilde{A}_{\Gamma}$ is positive and large. Indeed, the electrostatic term in $U$ induce a shift in the frequency of the zone-center optical modes which become longitudinal rather than transverse, and the LO-TO splitting in perovskite titanates is enormous ${ }^{26}$ As $\tilde{A}_{\Gamma}$ is positive, the only term in the Hamiltonian that can induce a polarization in open-circuit is precisely the trilinear one $C \phi_{z o} \phi_{z i} \xi_{\Gamma}$. Furthermore, as $\tilde{A}_{\Gamma}$ is large, $\xi_{\Gamma}$ will be virtually suppressed from the lowenergy Hamiltonian, and only a simultaneous presence of $z i$ and $z o$ rotations (at $\xi_{\Gamma}=0$ ) will produce a geometric field. In order to verify this possibility, we need to study the energy as a function of the AFD modes amplitude, i.e. the energy landscape that is sketched in Fig. 3.

\begin{tabular}{ccccc}
\hline \hline$A_{o}$ & $A_{i}$ & $B_{o o}$ & $B_{i i}$ & $B_{i o}$ \\
\hline-2.1097 & -0.6912 & 0.0368 & 0.0363 & 0.1372 \\
\hline \hline
\end{tabular}

TABLE II: Parameters of the model described in the text. Values of the parameters are in $\mathrm{eV}$. The independent variables $\phi$ are in degrees.

In absence of $\xi_{\Gamma}$, the low-energy Hamiltonian reduces to

$$
\begin{aligned}
& E_{\mathrm{KS}}\left(\phi_{z o}, \phi_{z i}\right)=A_{o} \phi_{z o}^{2}+A_{i} \phi_{z i}^{2}+ \\
& +B_{o o} \phi_{z o}^{4}+B_{i i} \phi_{z i}^{4}+B_{i o} \phi_{z o}^{2} \phi_{z i}^{2} .
\end{aligned}
$$

The values of the five parameters are reported in Table III the maximum deviations with the actual firstprinciples data are lower then $1.5 \mathrm{meV}$ across the whole two-dimensional energy landscape.

The necessary and sufficient condition for having four minima where $\phi_{z o}$ and $\phi_{z i}$ are nonzero is that the "pure" minima (where only one degree of freedom is active at the time) are in fact saddle points of the two-dimensional function $E\left(\phi_{z o}, \phi_{z i}\right)$,

$$
\begin{aligned}
& A_{o}-\frac{A_{i} B_{i o}}{2 B_{i i}}<0 \\
& A_{i}-\frac{A_{o} B_{i o}}{2 B_{o o}}<0
\end{aligned}
$$

It is easy to see that the first of these conditions is satisfied, i.e. the $\mathrm{AFD}_{\mathrm{zi}}$ configuration is not a true minimum but a saddle point in this reduced configuration space. Conversely, the second condition is violated, i.e. the pure $\mathrm{AFD}_{\mathrm{zo}}$ state is indeed a global minimum, where the original in-phase AFD instability is suppressed.

\section{DISCUSSION}

In the previous section we have clarified the physical ingredients that are responsible for the calculated electrical behavior of $\mathrm{YMnO}_{3}$ and $\mathrm{PTO} / \mathrm{STO}$. However, a substantial limitation of our work so far has been the complete neglect of temperature effects. As the concept of "improper ferroelectric" is intimately bound to the thermodynamics of phase transitions, it is useful to discuss here whether we can infer a number of likely scenarios based on our zero-temperature results. (Of course, our arguments will remain limited to a qualitative level, as presently we don't have access to finite-temperature simulations.) This point is particularly important in order to reconcile our findings (that a true "geometric field" not present in $\mathrm{PTO} / \mathrm{STO}$ ) with the experimental data of Ref. 10, where the typical signatures of improper ferroelectricity emerge clearly. Given the relevance of this system in the development of novel multifunctional materials, we shall focus the following discussion on PTO/STO. 
Experimentally, PTO/STO undergoes a phase transition at $T_{C}=500 \mathrm{~K}$ from a high-temperature paraelectric state to a polar state where the spontaneous $P$ that increases linearly with decreasing $T$. To rationalize this phase transition, several scenarios are possible, depending on the respective behavior of the three active degrees of freedom. First, we can imagine a transition where the AFD modes act as primary order parameters in the transition, and the polarization emerges as a secondary effect; this is the classic picture of improper ferroelectricity. This, however, does not appear consistent with our simulations. In absence of polarization, the two AFD modes are mutually exclusive, and they don't break inversion symmetry alone. In other words, if we suppress $P$ there is no "geometric field" in the system. Second, we can imagine a transition where $\xi_{\Gamma}$ is the primary order parameter, and the AFD distortions are secondary. This is, however, inconsistent with the experiments of Ref. 10: in a second-order phase transition involving only $P$, one would expect $P \propto \sqrt{T_{C}-T}$, which differs from the reported linear behavior.

Interestingly, a third possibility was proposed in a recent work, 27 where analogous Hamiltonians involving a trilinear coupling were studied in a phenomenological framework. The scenario described in Ref. 27 is that of an "avalanche" first-order transition, where all three degrees of freedom condense simultaneously without any of them becoming unstable. This is a situation where the usual assumptions of Landau theory, and in particular the distinction between primary and secondary order parameters, break down: neither the polarization nor the AFD modes would act as either master or slave. This possibility can be simply understood by constructing a "reaction coordinate" $Q$ and by imposing that all three degrees of freedom must be proportional to $Q$. Then the Hamiltonian reduces to the following expression

$$
E_{\mathrm{KS}}(Q)=\alpha Q^{2}+\beta Q^{4}+\gamma Q^{3} .
$$

All the degrees of freedom are individually stable above the transition, so $\alpha$ is positive, and its magnitude will decrease with $T$. $\beta$ is necessarily positive at all temperatures, or thermodynamic stability is lost. One can easily see that if $\gamma$ is different from zero a secondary minimum will appear for sufficiently low $T$ (small $\alpha$ ), and will drop below the main minimum at $Q=0$ before the $Q=0$ minimum becomes a saddle point. Thus, in presence of this trilinear term in the Hamiltonian, the transition is very likely to be first-order and involve all degrees of freedom simultaneously.

Strictly speaking, even this hypothesis does not constitute an ideal match to the experimental results. A first-order phase transition necessarily involves a jump in the polarization from zero to a finite value at $T_{C}$, while a continuous curve was reported experimentally. One can imagine, however, that the transition might be only weakly first-order, and the discontinuity might be too small to be detectable in the experimental setup of Ref. 10. Another possibility is that the depolarizing field produced by the electrodes might have an impact on the measured $P$, or more generally that other degrees of freedom not considered here (e.g. nanodomains) might play a role in the transition. A last alternative is that standard DFT calculations do not properly describe the relative strength of the different instabilities although these methods have been successfully applied to many cases so far.

Resolving these issues goes beyond the scopes of the present work; what we want to stress here is that the electrical analysis used in this work constitutes a powerful tool to study complex ferroelectric systems where many degrees of freedom interact nontrivially with the polarization.

\section{CONCLUSIONS}

In conclusion, we have shown that constrained- $D$ calculations provide a powerful method to understand the electrical behavior of multifunctional oxide compounds. As practical examples, we discussed two different material systems: A prototypical improper ferroelectric, $\mathrm{YMnO}_{3}$, and short-period PTO/STO superlattices. Concerning $\mathrm{YMnO}_{3}$, our results have evidenced a characteristic "kink" in the electrical equation of state (energy $U$ as a function of the electric displacement $D$ ), which is responsible for the remarkable insensitivity of this material to the electrical boundary conditions $\frac{16}{\underline{16}}$ Concerning PTO/STO, our results indicate that the electrical properties depends crucially on the interaction between the two non-polar AFD modes: Only if the AFD modes are the dominant instabilities and they do not compete with each other we expect the electrical properties to be qualitatively similar to those of $\mathrm{YMnO}_{3}$. While our PTO/STO model does not appear to satisfy either condition, recent results 12 give strong indications for such a scenario to occur in a related material, $\mathrm{Ca}_{3} \mathrm{Mn}_{2} \mathrm{O}_{7}$. To illustrate the consequences of these findings in a realistic capacitor configuration, we showed how the $U(D)$ curve can be readily converted into a $P\left(\lambda_{e f f} / N\right)$ curve, where $\lambda_{e f f}$ is the effective screening length of the electrode interface and $N$ is the thickness.

As an outlook, Ref. 10 has introduced us to the remarkable possibility that two AFD rotation distortions can induce ferroelectricity via a trilinear coupling with the polarization. This idea holds much promise to realize novel multifunctional phenomena, and has led to the discovery of an entirely new class of "hybrid" improper ferroelectrics 28 . While rationalizing these breakthroughs by putting them on firm theoretical grounds, our work also illustrates some interesting new aspects of these systems that might be useful for future applications. For example, it appears clear from our data that, in contrast to $\mathrm{YMnO} 3$, in PTO/STO the AFD modes are very strongly coupled to the polarization. This implies that the control of octahedral rotations by means of an applied field is indeed possible, and the constrained- $D$ method appears 
as the ideal theoretical tool to investigate (and engineer) this functionality in a variety of oxide systems.

\section{Acknowledgments}

We acknowledge discussions with K.M. Rabe and J. Íniguez. This work was supported by the DOEBES under Award Number DE-SCOO02334 (CJF); by
DGI-Spain through Grants No. MAT2010-18113 and No. CSD2007-00041 (MS); by the European Commission through the project EC-FP7, Grant No. NMP3SL-2009-228989 "OxIDes" (PhG and MS); and by the Francqui Foundation through a Research Professorship $(\mathrm{PhG})$. We thankfully acknowledge the computer resources, technical expertise and assistance provided by the Red Española de Supercomputación (RES) and by the Supercomputing Center of Galicia (CESGA).
1 S. Bhattacharjee, E. Bousquet, and P. Ghosez, Phys. Rev. Lett. 102, 117602 (2009).

2 T. Günter, E. Bousquet, A. David, P. Boullay, P. Ghosez, W. Prellier, and M. Fiebig, Phys. Rev. B 85, 214120 (2012).

3 N. A. Hill, J. Phys. Chem. B 104, 6694 (2000).

4 A. P. Levanyuk and D. G. Sannikov, Sov. Phys. Usp. 17, 199 (1974).

${ }^{5}$ C. J. Fennie and K. M. Rabe, Phys. Rev. Lett. 97, 267602 (2006).

6 Y. Tokura, J. Magn. Magn. Mater. 310, 1145 (2007).

7 S.-W. Cheong and M. Mostovoy, Nature Materials 6, 13 (2007).

8 C. J. Fennie and K. M. Rabe, Phys. Rev. B 72, 100103 (2005).

9 T. Choi, Y. Horibe, H. T. Yi, Y. J. Choi, W. Wu, and S.-W. Cheong, Nature Materials 9, 253 (2010).

10 E. Bousquet, M. Dawber, N. Stucki, C. Lichtensteiger, P. Hermet, S. Gariglio, J.-M. Triscone, and P. Ghosez, Nature 452, 732 (2008).

11 J. M. Rondinelli and C. J. Fennie, Advanced Materials 24, 1961 (2012).

12 N. A. Benedek and C. J. Fennie, Phys. Rev. Lett. 106, 107204 (2011).

13 M. Stengel, N. A. Spaldin, and D. Vanderbilt, Nature Physics 5, 304 (2009).

14 P. E. Blöchl, Phys. Rev. B 50, 17953 (1994).

15 H. J. Monkhorst and J. D. Pack, Phys. Rev. B 13, 5188
(1976).

16 N. Sai, C. J. Fennie, and A. A. Demkov, Phys. Rev. Lett. 102, 107601 (2009).

17 M. Stengel and N. A. Spaldin, Phys. Rev. B 73, 075121 (2006).

18 O. Diéguez and D. Vanderbilt, Phys. Rev. Lett. 96, 056401 (2006).

19 O. Diéguez and D. Vanderbilt, Phys. Rev. B 76, 134101 (2007).

20 J. Junquera and P. Ghosez, Nature 422, 506 (2003).

21 M. Stengel, D. Vanderbilt, and N. A. Spaldin, Nature Materials 8, 392 (2009).

22 M. Stengel, D. Vanderbilt, and N. Spaldin, Phys. Rev B 80, 224110 (2009).

23 T. Kopp and J. Mannhart, J. Appl. Phys. 106, 064504 (2009).

24 A. K. Tagantsev, Ferroelectrics 69, 321 (1986).

25 J. Hong and D. Vanderbilt, Phys. Rev. B 84, 115107 (2011).

26 W. Zhong, R. D. King-Smith, and D. Vanderbilt, Phys. Rev. Lett. 72, 3618 (1994).

27 I. Etxebarria, J. M. Perez-Mato, and P. Boullay, Ferroelectrics 401, 17 (2010).

28 N. A. Benedek, A. T. Mulder, and C. J. Fennie, Journal of Solid State Chemistry pp. - (2012), URL http://www.sciencedirect.com/science/article/pii/S0022459612 